\section{Estudo da}

reprodutibilidade

das emissões

otoacústicas em

individuos normais

\author{
Mariana C. Guedes ${ }^{1}$, Simone N. Passos ${ }^{1}$, \\ Maria Valéria S. Goffi Gomez ${ }^{2}$, \\ Ricardo Ferreira Bento ${ }^{3}$
}

\section{Repeatability of evoked otoacoustic emissions in normally heariing adulits}

Resumo / Summary

I

ntrodução: alguns estudos relatam a importância da aplicação clínica no acompanhamento da função coclear em tratamentos com medicações ototóxicas ou em exposição a agentes nocivos à cóclea. Pesquisas sobre a reprodutibilidade das emissões otoacústicas foram realizadas para validar sua utilização na monitorização da função coclear, entretanto a experiência clínica nos mostra que os valores de variação entre teste-reteste não coincidem com os descritos na literatura. Objetivo: verificar a variação da amplitude de resposta das emissões otoacústicas transientes evocadas (TEOAEs) e das emissões otoacústicas por produto de distorção (DPOAEs). Forma de estudo: clínico prospectivo randomizado. Material e método: Foram estudados 10 indivíduos jovens com audição normal, usando programa Celesta 503, Madsen. Foram realizadas três avaliações com no mínimo três semanas de intervalo, mantendo-se constantes as variáveis clinicamente controláveis, tais como, tamanho da oliva, número de apresentações e intensidade do estímulo. Resultados: mostram que a amplitude das TEOAEs variou menos do que das DPOAEs, e que a amplitude da resposta inicial não mostrou ser determinante na variabilidade da resposta.

\footnotetext{
Fonoaudióloga Clínica. Ex-aluna do Curso Teórico-prático em Audiologia Clínica da Divisão de Clínica Otorrinolaringológica do HCFMUSP.

${ }^{2}$ Fonoaudióloga do Setor de Fonoaudiologia da Divisão de Clínica Otorrinolaringológica do HCFMUSP, Doutora em Ciências dos Distúrbios da Comunicação Humana pela UNIFESP/EPM

3 Professor Associado da Disciplina de Otorrinolaringologia da FMUSP.
}

Trabalho realizado na Divisão de Clínica Otorrinolaringológica do Hospital das Clínicas da Faculdade de Medicina da Universidade de São Paulo. Endereço para correspondência: A/C Maria Valéria S. Goffi Gomez - Av. Dr. Enéas Carvalho de Aguiar nº 255 Clínica Otorrinolaringológica do HCFMUSP 6º Andar - ICHC Artigo recebido em 10 de outubro de 2001. Artigo aceito em 06 de novembro de 2001. 


\section{INTRODUÇÃO}

As emissões otoacústicas (OAEs) são sons gerados dentro da cóclea normal, tanto espontaneamente ou como resposta a uma estimulação acústica (Norton e Stover, 1994). As primeiras respostas da cóclea foram captadas por David Kemp do Institute for Laryngology and Otology (ILO) em Londres, em 1978 (Kemp, 1978).

Vários estudos confirmam a origem coclear das emissões otoacústicas. Alguns apontam que as emissões otoacústicas podem ser medidas quando a resposta neural está ausente (Robinette e Durrant, 1997), outros que as emissões estão ausentes quando a cóclea é agredida por drogas ototóxicas (Zorowka e col., 1993) ou hipóxia (Lonsbury-Martin, 1987). Assim, embora ainda existam várias questões sem resposta a respeito da função coclear e as emissões otoacústicas, existe um crescente interesse na pesquisa das emissões como uma forma rápida, precisa e não-invasiva da função coclear (White e col., 1993; Norton e Stover, 1994).

Existem dois tipos básicos de emissões otoacústicas resultantes das atividades do ouvido interno: as espontâneas, que são aquelas que podem ser captadas sem apresentação de estímulo sonoro e as evocadas, que são aquelas obtidas secundariamente à presença de um som. Estas se dividem em emissões otoacústicas transientes evocadas (TEOAEs), emissões otoacústicas por produtos de distorção (DPOAEs) e emissões otoacústicas estímulofreqüência, ainda sem utilização clínica.

As TEOAE são eliciadas por cliques e estão presentes em praticamente todas as orelhas saudáveis. As TEOAE têm uso clínico em triagem auditiva, diagnóstico de alterações retrococleares e monitoramento da função coclear (Gattaz e Cerruti, 1994; Bento e col., 1998; Choi e col., 1999).

As emissões otoacústicas evocadas produtos de distorção são uma energia acústica originária da cóclea pela interação não-linear de dois tons puros aplicados simultaneamente e assim podemos analisar a atividade colear em freqüências específicas. As emissões otoacústicas evocadas transientes são respostas produzidas pela atividade das células ciliadas externas dentro da cóclea provocadas por um estímulo sonoro muito breve ( $c l i c k$ ) e representam uma resposta global da cóclea (Lopes Fo. e col., 1996; Hussain e col., 1998; Hotz e col., 1994).

Quando todos os níveis de audição de $250 \mathrm{~Hz}$ a $8 \mathrm{KHz}$ são melhores que $20 \mathrm{~dB}$, as TEOAE estão presentes em 99\% das orelhas. A resposta TEOAE é composta de componentes de múltiplas fontes, e estas interagem de maneira complexa para fornecer o resultado final (Harris e Probst, 1997).

As emissões otoacústicas são transmitidas da cóclea através dos ossículos e membrana timpânica (MT) e medidas no conduto auditivo externo (CAE). Qualquer alteração de orelha média (OM) ou obstrução do CAE podem potencialmente impedir a transmissão das TEOAE. Portanto, é recomendado que a testagem de TEOAE seja complementada com imitanciometria (Choi e col., 1999). Limiares superiores a $30 \mathrm{~dB}$ (NA) não mostram presença de emissões otoacústicas transientes, tanto pela falha na função das células ciliadas externas como pela falta de intensidade suficientemente forte para estimulá-las. Por outro lado, em perdas auditivas até $45 \mathrm{~dB}$ (NA) é possível a obtenção das respostas por produto de distorção (Miniti e col., 2000).

Desde sua descoberta, a pesquisa das OAEs é largamente usada em triagem neonatal, como auxiliar no diagnóstico de alterações neurais, no acompanhamento da função coclear em tratamentos com medicações ototóxicas ou em exposição a agentes nocivos à cóclea (Robinette e Glattke, 1997).

Alguns autores estudaram a reprodutibilidade das emissões otoacústicas para validar sua utilização no monitoramento da função coclear (Harris e col., 1991; Vedantam e Musiek, 1991; Franklin e col., 1992; Roede e col., 1993), entretanto a experiência clínica nos mostra que os valores de variação entre teste-reteste não coincidem com os descritos na literatura.

A presente pesquisa pretende estudar e quantificar as variações individuais da amplitude relativa das emissões otoacústicas transientes evocadas e produto de distorção, em indivíduos normais.

\section{MATERIAL E MÉTODO}

Esta pesquisa foi realizada no setor de fonoaudiologia da Divisão de Clínica Otorrinolaringológica do Hospital das Clínicas da Faculdade de Medicina da Universidade de São Paulo. Foram analisados os resultados das emissões otoacústicas transientes evocadas (TEOAEs) e por produto de distorção (DPOAEs) de 10 indivíduos sem alterações auditivas e sem passado otológico, sem exposição a ruído ocupacional e na ausência de tratamentos com drogas ototóxicas, com idade variando entre 22 e 38 anos de idade.

Para participar do estudo, todos os indivíduos deveriam apresentar limiares auditivos normais (até $25 \mathrm{~dB}$ NA em todas as freqüências testadas, de $250 \mathrm{~Hz}$ a $8000 \mathrm{~Hz}$ ), curvas timpanométricas normais e presença de reflexos estapedianos até $100 \mathrm{~dB}$ NA, em todas as freqüências testadas ( $500 \mathrm{~Hz}$ a $4000 \mathrm{~Hz}$ ), em ambas as orelhas.

A determinação dos limiares foi realizada pelo método descendente no audiômetro Madsen Midimate 622. Para a imitanciometria foi utilizado equipamento Interacoustics AZ 26.

As emissões otoacústicas foram realizadas em sala acusticamente tratada, com equipamento CELESTA 507, Madsen. 
Cada indivíduo foi submetido a três pesquisas das TEOAEs e das DPOAEs, com intervalo de quatro semanas entre cada avaliação.

Antes do início de cada teste foi verificada a posição e estabilidade da sonda (check fit). As TEOAEs foram realizadas com estímulo não-linear, a 80 dB, com 1000 amostras. O critério para a presença das respostas foi considerado como uma correlação de, no mínimo, 0,50 entre as respostas (reprodutibilidade) e amplitude da resposta global de no mínimo $3 \mathrm{~dB}(\mathrm{~S} / \mathrm{N} \geq 3 \mathrm{~dB})$.

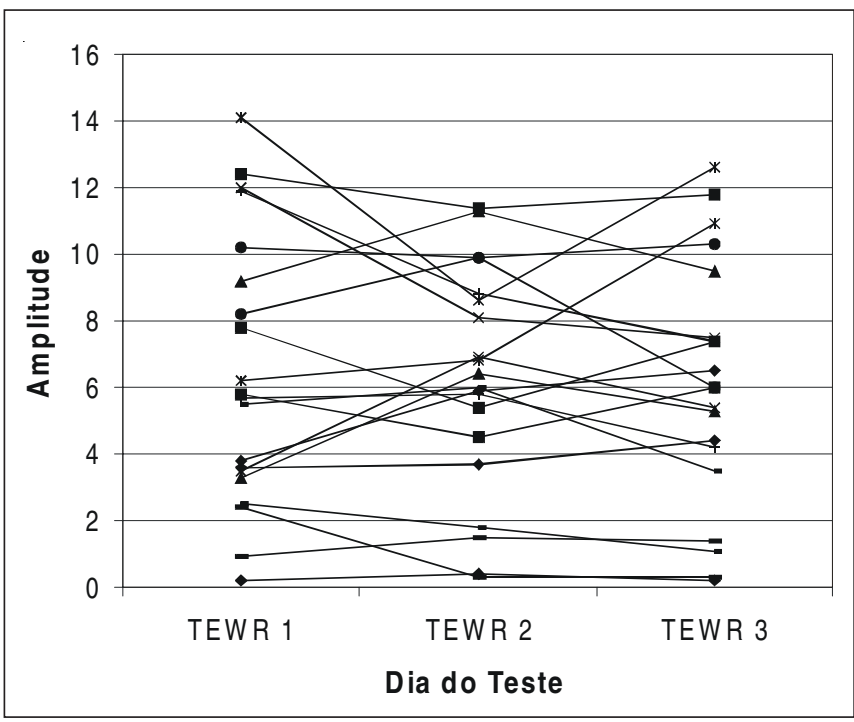

Gráfico 1. Respostas obtidas das 20 orelhas testadas em cada uma das três avaliações.
As DPOAEs foram realizadas com estímulos $\mathrm{f} 1 / \mathrm{f} 2$ a $70 \mathrm{~dB}$ (relação de 1,2), sendo o exame finalizado ao atingir $100 \mathrm{~ms}$ ou $12.0 \mathrm{~dB}$ de amplitude por freqüência. Foram analisadas as respostas nas freqüências de $1000 \mathrm{~Hz}, 2000$ Hz, 4000 Hz, 6000 Hz e 8000 Hz. A resposta de DP1 (2f1f2) foi considerada presente quando a amplitude da emissão ultrapassava $6 \mathrm{~dB}$ o nível de ruído $(\mathrm{S} / \mathrm{N} \geq 6 \mathrm{~dB})$.

Foram mantidas constantes as condições de teste passíveis de controle, tais como: tamanho de oliva, número de apresentações e intensidade.

\section{RESULTADOS}

Foi realizada a análise da variação das respostas entre cada teste realizado, com o intervalo de quatro semanas. A variação média, em dB, entre cada teste está representada na Tabela 1 , tanto para as emissões otoacústicas transientes com para as emissões otoacústicas por produto de distorção em cada uma das freqüências testadas.

No Gráfico 1 pode-se observar a variação da amplitude de resposta das emissões otoacústicas transientes nos indivíduos pesquisados em cada um dos testes realizados.

$\mathrm{Na}$ análise dos resultados das emissões otoacústicas transientes a média de variação foi de 1,49dB (variando de 0 a 3,67, desvio padrão de 1,1 e mediana de 1,5). Na análise dos resultados das emissões otoacústicas por produto de distorção a variação média foi de $2,97 \mathrm{~dB}$ em $1000 \mathrm{~Hz}, 2,14 \mathrm{~dB}$ em $2000 \mathrm{~Hz}, 3,13 \mathrm{~dB}$ em $4000 \mathrm{~Hz}, 4,23 \mathrm{~dB}$ em $6000 \mathrm{~Hz}$ e $7,59 \mathrm{~dB}$ em $8000 \mathrm{~Hz}$, com desvios padrão de 2,$06 ; 1,19 ; 1,74 ; 1,94$ e 4,48 respectivamente. Os dados estão representados na Tabela 2 .

Tabela 1. Variação da amplitude das respostas das emissões transientes evocadas e por produto de distorção (em dB) nas diferentes frequências testadas nas três testagens realizadas.

\begin{tabular}{lcccccc}
\hline & Amplitude global (TE) & $1000 \mathrm{~Hz}(\mathrm{DP})$ & $2000 \mathrm{~Hz}(\mathrm{DP})$ & $4000 \mathrm{~Hz}(\mathrm{DP})$ & $6000 \mathrm{~Hz}(\mathrm{DP})$ & $8000 \mathrm{~Hz}(\mathrm{DP})$ \\
\hline R2-R1 & 1,56 & 2,60 & 2,16 & 3,14 & 4,50 & 8,68 \\
R3-R2 & 1,41 & 2,99 & 2,48 & 2,42 & 5,20 & 7,17 \\
R3-R1 & 1,50 & 3,29 & 1,79 & 3,83 & 3,37 & 7,73 \\
Variação média & 1,49 & 2,97 & 2,14 & 3,13 & 4,23 & 7,59 \\
\hline
\end{tabular}

Legenda: R2-R1: diferença entre a amplitude da resposta na segunda avaliação e na primeira avaliação. R3-R2: diferença entre a amplitude da resposta na terceira avaliação e na segunda avaliação. R3-R1: diferença entre a amplitude da resposta na terceira avaliação e na primeira avaliação.

Tabela 2. Valores mínimos e máximos de variação, média da melhora ou da piora das respostas das emissões transientes evocadas e por produto de distorção (em dB) nas frequências testadas entre todas as testagens realizadas.

\begin{tabular}{|c|c|c|c|c|c|c|}
\hline & Resposta global (TE) & $1000 \mathrm{~Hz}$ & $2000 \mathrm{~Hz}$ & $4000 \mathrm{~Hz}$ & $6000 \mathrm{~Hz}$ & $8000 \mathrm{~Hz}$ \\
\hline Menor variação & 0,00 & 0,60 & 0,20 & 0,40 & 1,40 & 2,20 \\
\hline Maior variação & 3,67 & 9,27 & 4,33 & 6,60 & 7,47 & 20,20 \\
\hline Média da diminuição da amplitude & 1,38 & 3,02 & 1,91 & 3,15 & 3,35 & 6,65 \\
\hline Média do aumento de amplitude & 0,88 & 2,88 & 2,34 & 3,11 & 4,48 & 8,72 \\
\hline
\end{tabular}




\section{DISCUSSÃO}

Provavelmente o benefício mais notável das OAEs é sua habilidade de inspecionar de forma objetiva e nãoinvasiva os primeiros estágios do processamento do som ao nível da atividade biomecânica das CCEs. É bem conhecida a importante susceptibilidade deste receptor celular aos efeitos adversos de doenças virais e bacterianas, alterações genéticas, agentes externos, tais como sons intensos, drogas ototóxicas e químicas, que danificam a audição. É a extraordinária propriedade seletiva e sensitiva de "localização da lesão" das respostas emitidas que encorajou os pesquisadores a desenvolver estes procedimentos em métodos de utilidade clínica para a abordagem dos estágios iniciais do processo auditivo (LonsburyMartin e col., 1995).

Para um processo de monitoramento da audição é necessária a determinação de parâmetros confiáveis sobre a variação das respostas em indivíduos normais para que os mesmos possam ser aplicados em casos de pacientes tratados com drogas ototóxicas, que façam uso de produtos químicos danosos ao sistema auditivo ou expostos a ruído elevado.

Em relação ao uso de drogas ototóxicas, Zorowka e col. (1993) observaram uma amplitude reduzida nas TEOAE de 08 crianças que faziam o uso de cisplatina em relação ao grupo controle e Hotz e col. (1994) encontraram amplitudes cerca de 3,2dB menores em indivíduos que faziam o uso de aminoglicosídeos (sulfato de amicacina) nas TEOAEs.

Franklin e col. (1992) encontraram uma variação de aproximadamente $2 \mathrm{~dB}$ na amplitude das TEOAE em indivíduos normais $(n=12)$. Na presente pesquisa as TEOAEs mostraram uma variação de $3.69 \mathrm{~dB}$ (considerando a média $+2 \mathrm{DP})$.

Harris e col. (1991) encontraram uma variabilidade de $2 \mathrm{~dB}$ em um estudo com 10 indivíduos normais usando o analisador ILO 88. Essa diferença poderia ser explicada pela utilização de programas diferentes na geração e captação das OAEs. Como os autores citados, nesta pesquisa também foi observada que a variabilidade das respostas não estão relacionadas com a amplitude das mesmas, ou seja, respostas pequenas continuaram pequenas e aquelas respostas com uma amplitude maior mantiveram-se semelhantes.

Vedantam e Musiek (1991) encontraram alta correlação entre teste-reteste nas OAE $(\mathrm{p}<0,0001)$, porém o intervalo entre os exames era de apenas uma hora e trinta minutos o que possibilitava um maior controle sobre variações do estado físico geral do indivíduo e condições do ambiente.

Sabe-se que alterações na pressão da orelha média podem causar diminuição na amplitude da resposta ou que a falta de estabilidade da oliva no conduto auditivo externo interferiria na captação das OAEs. Dessa maneira tentou-se ao máximo controlar os parâmetros durante a realização do teste e na obtenção das respostas, como ruído, condições de orelha média e colocação da oliva no conduto auditivo externo, contudo acreditamos que existam outros fatores que podem influenciar na captação das respostas e na amplitude das mesmas e que não são possíveis de serem controladas rigidamente pelo pesquisador em situação clínica.

A variação da amplitude relativa também foi analisada nas DPOAEs, sendo encontrada maior variação na amplitude nas freqüências agudas $(6000 \mathrm{~Hz}$ e $8000 \mathrm{~Hz})$ do que nas freqüências de $1000 \mathrm{~Hz}$ e $2000 \mathrm{~Hz}$, justamente as freqüências que seriam as inicialmente afetadas por agentes ototóxicos, químicos e por ruído e que precocemente mostrariam o início do envolvimento das células ciliadas externas. De acordo com os estudos de Roede e col. (1993), uma variação de 6 a 9dB na amplitude das DPOAE poderia indicar uma mudança significativa na dinâmica coclear desde que mantidas as condições da orelha média.

Franklin e col. (1992) encontraram uma variação média de $2 \mathrm{~dB}$ na amplitude das freqüências médias nas DPOAEs e uma variação maior que $2 \mathrm{~dB}$ nas freqüências extremas (1KHz e 8KHz). Littman e col. (1998) observaram ausência de resposta nas DPOAEs em um indivíduo após doses cumulativas de cisplatina antes da piora dos limiares na audiometria tonal.

Comparando as respostas das TEOAEs e DPOAEs verificou-se uma variabilidade menor da amplitude relativa das emissões otoacústicas transientes do que da amplitude das emissões obtidas por produto de distorção.

As respostas variaram tanto para melhor (aumento da amplitude) como para pior (diminuição da amplitude), porém nenhum dos indivíduos estudados apresentou amplitudes inferiores a $3 \mathrm{~dB}$ na TEAOAE ou $6 \mathrm{~dB}$ na DPOAE em qualquer das testagens.

De acordo com o observado, acreditamos ser de suma importância a análise de outros parâmetros e variáveis, como a amplitude absoluta e o nível de ruído em cada teste. Este estudo prossegue com a inclusão da análise desses parâmetros e o estudo estatístico em uma amostra maior.

\section{CONCLUSÃO}

Diante dos resultados do presente estudo sobre a variação da amplitude relativa de resposta das emissões otoacústicas em indivíduos normais podemos concluir que, na população estudada:

- As TEOAEs variaram menos que as DPOAEs em indivíduos normais;

- A variação média das TEOAEs foi de 3,69dB (2 DP;

- A menor variação das DPOAEs ocorreu na freqüência de $2000 \mathrm{~Hz}$ sendo 2,14 dB;

- A variação das DPOAEs foi maior nas freqüências de 6000 e $8000 \mathrm{~Hz}$. 


\section{REFERÊNCIAS BIBLIOGRÁFICAS}

1. BENTO, R.F.; MINITI, A.; MARONE, S. - Tratado de Otologia. São Paulo. Edusp. 1998 p. 107-16.

2. CHOI, S.S.; PAFITIS, I.A.; ZALZAL, G.H.; HERER, G.R.; PATERL, K.M. - Clinical Applications of Transiently evoked otoacoustic emissions in the pediatric population. Ann Otol Rhinol Laryngol 108:132-8. 1999.

3. ENGDAHL, B.; ARNESEN, R.; MAIR, I. - Reproducibility and shortterm variability of transient evoked otoacoustic emissions. Scandinavian Audiology 23:99-104. 1994

4. FRANKLIN, D.J.; McCOY, M.J.; MARTIN, G.K.; LONSBURY-MARTIN, B.L. - Test-retest reliability of distortion-product and transiently evoked otoacoustic emissions. Ear and Hearing 13 (6):417-29. 1992.

5. GATTAZ, G.; CERRUTI, V.Q. - O Uso do Registro de Emissões Otoacústicas Evocadas para Triagem Auditiva em Neonatos de Risco para Deficiência Auditiva. Revista Paulista de Pediatria 12 (3):291-4. setembro 1994.

6. HARRIS, F.P.; PROBST R. - Otoacoustic Emissions and Audiometric Outcomes. IN: ROBINETTE, M.S. e GLATTKE, T.J. Otoacoustic Emissions. Clinical Applications. Thieme. New York. 1997. Cap. 8.

7. HARRIS, F.P.; PROBST, R.; WENGER, R. - Repeatability of transiently evoked otoacoustic emissions in normally hearing humans. Audiology 30:135-41. 1991

8. HOTZ, M.A. HARRIS, F.P. E PROBST, R. - Otoacoustic Emissions: An Approach for Monitoring Aminoglycoside-Induced Ototoxicity. Laryngoscope 104:1130-4. 1994.

9. HUSSAIN, D.M.; GORGA, M.P.; NEELY, S.T.; KEEFE, D.H.; PETERS, J. - Transient Evoked Otoacoustic Emissions in Patients with Normal Hearing and in Patients with Hearing Loss. Ear Hear. 434-49. 1998.

10. KEMP, D.T. - Development in cochlear mechanics and techniques for noninvasive evaluation. Advanced Audiology 5:27-45. 1988.

11. KEMP, D.T. - Stimulated acoustic emissions from within the human auditory system. Journal of the Acoustic Society of America 64:1386-91. 1978.

12. LITTMAN, T.A.; MAGRUDER, A.; STROTHER, D.R. - Monitoring and predicting ototoxic damage using distortion-product otoacoustic emissions: Pediatric case study. J. Am Acad Audiol 9:257-62. 1998.
13. LONSBURY-MARTIN, B..L. - Acoustic distortion products in rabits. I. Basic features and physiological vulnerability. Hear Res 28:173-6. 1987.

14. LONSBURY-MARTIN, B..L.; MARTIN, G.K.; MC COY, M.J.; WHITEHEAD, M.L. - New approaches to the evaluation of the auditory system and a current analysis of otoacoustic emissions. Otoalryngol Head Neck Surg 112:50-63. 1995.

15. LOPES FILHO, O.; CARLOS, R.; THOMÉ, D.; ECKLEY, C. - Emissões Otoacústicas Transitórias e Produtos de Distorção na Avaliação da Audição em Recém-Nascidos com Poucas Horas de Vida, Revista Brasileira de Otorrinolaringologia 62 (3):220-8. 1996

16. MINITI, A.; BENTO, R.F.; BUTUGAN, O. - Otorrinolaringologia Clínica e Cirúrgica. $2^{2}$ edição. São Paulo. Atheneu. 2000. Cap.10.

17. NORTON, S.J. e STOVER, L.J. - Otoacoustic Emissions: An emerging tool. IN: KATZ, J. Handbook of Clinical Audiology. $4^{\text {th }}$ edition. William and Wilkins. Baltimore. 1994.

18. ROBINETTE, M.S. e DURRAN, J.D. - Contributions of evoked otoacoustic emissions in differential diagnosis of retrococlear disorders. IN: ROBINETTE, M.S. e GLATTKE, T.J. Otoacoustic Emissions. Clinical Applications. Thieme. New York. 1997.

19. ROBINETTE, M.S. e GLATTKE, T.J. - Otoacoustic Emissions. Clinical Applications. Thieme. New York. 1997.

20. ROEDE, J.; HARRIS, F. P.; PROBST, R; XU, L. - Repeatability of distortion product otoacustic emissions in normally hearing humans. Audiology 32:273-81 1993.

21. VEDANTAM, R.; MUSIEK, F.E. - Click evoked otoacoustic emissions in adult sugjects: standard indices and test-retest reliability. Am J Otology 12 (6):485-442. 1991.

22. WHITE, K.R.; VOHR, B.R.; BEHRENS, T.R. - Universal Newborn Hearing Screening Using transient evoked otoacoustic emissions: Results of the Rhode Island Hearing Assessment Project. Seminars in Hearing 14 (1):18-29. 1993.

23. ZOROWKA, P.G.; SCHMITT, H.J.; GUTJAHR, P. - Evoked otoacoustic emissions and pure tone threshold audiometry in patients receiving cisplatinum therapy. Int J Pediatric Otorbinolaryngol 25:73-80. 1993. 